\title{
Which are the Molecular Basis of Dysplasia Type IIIa, What Should We Know and Why?
}

\section{René Andrade-Machado}

National Institute Neurology, CES University, Colombia

Corresponding author: René Andrade-Machado, National Institute Neurology, CES University, Colombia, Tel: 57 (4) 576 66 66; E-mail: reneandrade1970@yahoo.es

Received date: July 29, 2016; Accepted date: July 30, 2016; Published date: Aug 02, 2016

Copyright: (c) 2016 Andrade-Machado R. This is an open-access article distributed under the terms of the Creative Commons Attribution License, which permits unrestricted use, distribution, and reproduction in any medium, provided the original author and source are credited.

\section{Editorial}

Temporal lobe epilepsy is the most frequent epileptic syndrome in adult patients with epilepsy. About $70 \%$ of patients who suffer from this type of epileptic syndrome do not respond to antiepileptic medications. Thus, most of the patients are carried out to anteromesial temporal lobectomy in order to achieve control of their seizures [1].

In specimens taken from temporal lobe in these patients, Hippocampal Sclerosis (HS) is found in the vast majority of the cases. Around $76.3 \%$ of patients have also some kind of cortical dyslamination or abnormal cortical neuronal orientation in the temporal neocortex. According to ILAE consensus published in 2010, this features should better called dysplasia type IIIa [2].

Many papers are focused in the risk factors associated to temporal lobe epilepsy with HS. Complex febrile seizures, early head traumas, central nervous system infections or mild perinatal hypoxia have been associated with HS [3]. Although not totally elucidated, the molecular basis of HS is better known than the molecular basis of dysplasia type Ia or Ib. Thus, less is known about the complex mechanisms that provoke HS with dysplasia type Ia or Ib (dysplasia type IIIa).

In this field there are many questions that remaining without answers: Why some patients develop dysplasia type IIIa and others do not? Which would be the mechanisms that cause pathological processes, dysplasia and HS? Are persons prone genetically to have dysplasia type Ia or Ib and these cortical cytoarchitectural changes make peoples vulnerable to the first insult? Are these pathological processes an independent phenomenon each other? What keep the cerebral cortex organization during postnatal life? Is the cortical organization a static or dynamic process where the vitality of hippocampal neurons is needed? Is dysplasia type IIIa an acquired process? Might both, dysplasia type IIIa and HS, share the same molecular basis?

To elucidate the molecular basis of dysplasia type IIIa can help to understand most of the above mentioned questions and also may help to understand the evolution and progression of temporal lobe epilepsy. It might help to explain their clinical features and common comorbidity (depressive disorders, psychosis and amnesia). Otherwise, the knowledge of molecular basis of dysplasia type IIIa is very important to find different biomarkers, biomarker of epileptogenicity, epileptogenesis, progression's markers, and even markers of diseases resolution. As important as the above mentioned implications, molecular basis let to scientific community to find possible targets for the treatment of epilepsy and to find some preventive strategies.

Hippocampal sclerosis is characterized by important cell loss in CA2, CA4, CA3, astrogliosis and severe sprouting process [4], whereas dysplasia type Ia and $\mathrm{Ib}$ are characterized by an abnormal cortical lamination that can be tangential or horizontal [4]. A unifying theory should be able to explain all of these features, cell loss, sprouting, gliosis and the cortical cytoarchitectural abnormalities.

According to the most recent publications there are some hypotheses that revels some insights about the possible mechanisms of dysplasia type IIIa, they include, although are not limited to, the reelin, adenosine and the Wnt/b-catenin hypothesis.

Nowadays the adenosine system has been considered a powerful anticonvulsant and neuroprotective system. During a spontaneous seizure, the level of $\mathrm{A}$ is increased and as consequence the seizures are auto limited. Experimental studies have shown total control of refractory seizures after A had added. Adenosine is thought to stimulate presynaptic and postsynaptic receptors reducing the cell calcium imputes and the postsynaptic excitability, by increasing the outflows of potassium ions through the activation of the called potassium rectifying channels [5].

It is known that the over expression of Adenosine Kinase (ADK) cause A deficiency. The astrogliosis, a common feature found in HS, increase the levels of ADK. The identification of the relationship of the over expression of ADK and recurrent seizures was firstly reported in a mouse model of temporal lobe epilepsy [6].

The injection of kainic acid in one of the basso-lateral nuclei of the amygdala in mousses causes reactive astrogliosis only in the lateral aspect of the injected amygdala and CA3 region of the ipsilateral hippocampus. In these cases intracranial recordings only showed recurrent, refractory seizures arisen from the site of astrogliosis. Interestingly, the seizures appeared synchronically to the developing of the astrogliosis and the over expression of ADK [6].

Is the astrogliosis itself or the over expression of ADK what causes recurrent and refractory seizures?

Transgenic modified mousses clarified this question. Transgenic mousses in which the gen codifying for ADK enzyme was over expressed, suffer from recurrent and refractory seizures. These seizures appeared even if they would not be induced by kainic acid injection. This investigation revealed that the over expression of ADK induces recurrent and refractory seizures. Most important results were found when the investigators obtained transgenic modified mousses that did not express ADK. In this model, the mouse did not suffer from seizures [6].

It is thought that the over expression of ADK is a primary and no a secondary factors of epileptogénesis due to the recurrent seizures do not provoke over expression of ADK [6].

The amnesic symptoms found in patients with temporal lobe epilepsy can be explained by the adenosine hypotheses. Some studies 
have shown that mousses with over expression of ADK have less spatial recognition, spatial orientation and spatial memory. This suggests that the over expression of ADK would explain the typical cognitive deficits found in temporal lobe epilepsy [6].

\section{The reeline hypothesis}

Reelin is a glycoprotein that acts as stopping migration signal. The mutation of reelin in mousses cause a special phenotype characterized by an abnormal radial and tangential neuronal orientation in the cerebral cortex [7]. This feature prone to think that the absence of reelin could be involved in the pathogenesis of dysplasia type Ia or Ib.

In adults brain the reelin is secreted by interneurons. Reelin contributes to homeostasis of amino acids, such as glutamate, and it is necessary to achieve a normal composition of NMDA receptors in hippocampal neurons. Low levels of reelin have been associated to dispersion of granular cell in the hippocampus of patients with temporal lobe epilepsy. Low concentration of mRNA codifying for reelin have been associated with high level of granular dispersion [8].

The reelin deficiency determines a structural reorganization that increased the migratory activity of the new born neurons and abnormal dendrite plasticity. Thus, reelin is involved in the maintaining of the structural organization of cerebral cortex [7].

To test the reelin hypothesis, 30 specimens of patients underwent temporal lobectomy because uncontrolled seizures were studied. Immunohistochemical studies included antibody against $\mathrm{Neu}$ and reelin. All patients showed dysplasia type Ia or Ib, 15 patients had dysplasia type I (HS without granular cell dispersion (8 cases), and HS with granular cell dispersion type I (7 cases)) (group I), on the other hand, 15 patients had dysplasia type I and granular cell dispersion type 2 (group II). Positive reelin neurons were found in the $53.3 \%$ of cortical neurons and in the 86 , and $6 \%$ of hippocampal neurons, whereas only $20 \%$ of the cortical neurons and $13.4 \%$ of hipocampal neurons were positive to reelin I group II [9]. According to the above mentioned results reelin is associated with granular cell dispersion and abnormal cortical lamination.

One of the most important mechanisms that cause excitability and abnormal inhibition within hippocampus is the generation of abnormal circuitries due to the abnormal sprouting occurring after the cell death [10].

The setting of abnormal branches and dendrites positioning requires the maintenance of an ongoing process called tissue remodeling. Reelin stimulates the actin associated protein called, n-cofillin. F actin binds $\mathrm{n}$-cofillin promoting disassembled of actins' filaments. This process is regulated by the LIM kinase activity. The phophorilation of $\mathrm{n}$-cofillin by LIM kinase avoid the disassembling of $\mathrm{F}$ actin. Two dependent reelin activation proteins (Dab-1 and IPK) are required to phophorilate $\mathrm{n}$-cofillin. Thus, reelin is necessary to stabilize the cell cytoskeleton and the leading process, to guide neurons' migration. Investigations using mousse models, knockout to the n-cofillin gen, have showed that the mousses develop abnormal cortical organization. This feature supports the hypothesis of the role of $n$-cofillin pathway in cortical organization [11].

What cause the reduction of reelin turnover? An increased of tyrosine kinase $(\mathrm{TrKb})$ activity, the high affinity of reelin for BDNF (brain derive neurotrophic factor) and an increased of metilation of reelin gen [12]. It is known that maternal deprivation, depressive disorders, stress, central nervous system infections and hyperthermia have been related to an increase of DNA metilation [13,14]. Also the over expression of $\mathrm{ADK}$ and low intracellular adenosine concentrations have been associated with DNA abnormal mentilation [6]. Thus, these factors can be related to an abnormal cortical organization, dispersion of granular cells, HS.

Other feature found in temporal lobe epilepsy is a dysfunction in neurogénesis. The Wingless-related integration site (WNT/bcathenine) pathways have been involved in the signaling pathway related to neurogenesis. This pathway has an important role in proliferation, neuronal migration, cellular polarity and central nervous system homeostasis [15].

Recently publications have emphasized the role of Wnt/b-cathenin pathways in epileptogenesis. Mouse epileptic model using pilocarpine as inductor of status epilepticus showed a severe cell loss in CA3 and $\mathrm{CA} 1$ regions, and b-cathenine increased in the pyramidal layer and abnormal sprouting in the mousses hippocampus. Increased levels of WNT-2 and b-cathenine in dentate gyrus have been found after electroconvulsive therapy in mousses models of epilepsy. Experimental studies have found an over expression of b-cathenine, SFRP2, DVL3, Wnt-2, Wnt-5a y 10a genes after hypoxic injury [15].

One study showed the over expression of Dkk-1(an inhibitor of Wnt/b-cathenine pathway) in patient with temporal lobe epilepsy and HS. This study suggests the role of Dkk-1 in cell neurodegeneration [15].

Investigation in knockout mousses for the b-cathenine gen have showed a major susceptibility of theses mousses to have refractory seizures and long lasting seizures. Also, an abnormal differentiation of the cortical layer, cell loss in the CA1, 2, 3 hippocampus regions and in the dentate gyrus when were compared with controls. These features suggest that b-cathenine can pay a pivotal role in the cortical organization disorders like have been found dysplastic lesions [15].

Since dysplasia type IIIa was recognized by ILAE commission as a cause of temporal lobe epilepsy, few studies have been conducted to evaluate the mechanism involved in the pathogenesis. As we can see, there is a big gap in the current knowledge about pathogenic mechanisms related to the developing of dysplasia type IIIa. Nevertheless, reelin, adenosine and Wnt/-cathenin pathways can play a pivotal role in this intricate molecular mechanism. To figurate out the molecular basis of this disorder, may help to understand not only the basic mechanism of epilepsy, but also how to stop the epileptogenic mechanism. The problem of the epilepsy is not to investigate anticonvulsants anymore; it is to investigate medication that prevents the epileptogenic process. In order to do that, we should know what the molecular bases that cause this process are.

\section{References}

1. Schaller K, Cabrilo I (2016) Anterior temporal lobectomy. Acta Neurochir (Wien) 158: 161-166.

2. Wu J, Li W, Chen Y, Kang L, Zhao W (2015) Clinical characteristics of 92 patients with temporal lobe focal cortical dysplasia identified by pathological examination. J Clin Neurosci 21: 2170-2174.

3. Tatum WO (2012) Mesial temporal lobe epilepsy. J Clin Neurophysiol 29: 356-365.

4. Blumcke I, Thom M, Aronica E, Armstrong DD, Vinters HV, et al. (2011) The clinicopathologic spectrum of focal cortical dysplasias: A consensus classification proposed by an ad hoc Task Force of the ILAE Diagnostic Methods Commission. Epilepsia 52: 158-174.

5. Boison D (2012) Adenosine dysfunction in epilepsy. Glia 60: 1234-1243. 
Citation: Andrade-Machado R (2016) Which are the Molecular Basis of Dysplasia Type Illa, What Should We Know and Why?. Epilepsy J 2: e112. doi:10.4172/2472-0895.1000e112

Page 3 of 3

6. Boison D (2008) The adenosine kinase hypothesis of epileptogenesis. Prog Neurobiol 84: 249-262.

7. Frotscher M, Chai X, Bock HH, Haas CA, Förster E, et al. (2009) Role of Reelin in the development and maintenance of cortical lamination. J Neural Transm 116: 1451-1455.

8. Haas CA, Dudeck O, Kirsch M, Huszka C, Kann G, et al. (2002) Role for reelin in the development of granule cell dispersion in temporal lobe epilepsy. J Neurosci 22: 5797-5802.

9. Müller MC, Osswald M, Tinnes S, Häussler U, Jacobi A, et al. (2009) Exogenous reelin prevents granule cell dispersion in experimental epilepsy. Exp Neurol 216: 390-397.

10. Al Sufiani F, Ang LC (2012) Neuropathology of temporal lobe epilepsy. Epilepsy Res Treat 2012: 1-13.

11. Chai X, Zhao S, Fan L, Zhang W, Lu X, et al. (2016) Reelin and cofilin cooperate during the migration of cortical neurons: A quantitative morphological analysis. Development 1: 1029-1040.
12. Forster E, Bock HH, Herz J, Chai X, Frotscher M, et al. (2010) Emerging topics in Reelin function. Eur J Neurosci 31: 1511-1518.

13. Folsom TD, Fatemi SH (2013) The involvement of Reelin in neurodevelopmental disorders. Neuropharmacology 68: 122-125.

14. Maheu FS, Dozier M, Guyer AE, Mandell D, Peloso E, et al. (2010) A preliminary study of medial temporal lobe function in youths with a history of caregiver deprivation and emotional neglect. Cogn Affect Behav Neurosci 10: 34-49.

15. Busceti CL, Biagioni F, Aronica E, Riozzi B, Storto M, et al. (2007) Induction of the Wnt inhibitor, Dickkopf-1, is associated with neurodegeneration related to temporal lobe epilepsy. Epilepsia 48: 694-705. 